\title{
About the scientific contribution of Jean-Paul Malrieu
}

Published online: 3 August 2006

(c) Springer-Verlag 2006

\section{Early years...}

During the years 1965-1975 in Paris, there was a group of about ten fellows, all between 25 and 35 years old, wandering between "Pierre et Marie Curie" and "Lhomond" streets, busy with theoretical chemistry. "What an amazing situation", some people used to say! They are too numerous! We do not need so many workers in this peculiar field in this country! There will never be positions for them all! And why do they use computers? Are they too lazy and prefer computing rather than thinking?

Of course, within the group, the questions were more specific: Can we really trust gaussian functions? Is the four index transformation a bottleneck for ever? Is correlation negligible, important, critical and in what case? etc. And these questions were leading directly to deeper problems that were warmly debated in nearly daily sessions: Will theoretical chemistry be able some day to produce actual interpretations or predictions and not just to adjust ad hoc parameters or conclude that molecules are too complicated? Is it possible to develop theoretical approaches that avoid complicated numerical calculations? Or is it more efficient to look for simple numerical methods? What is more important: To develop general concepts which might explain trends but with always unexplained particular cases? Or just develop numerical methods able to find an interpretation in any specific case with no information on general trends? Is there anybody here

B. Lévy · Ph. Millié

Laboratoire de Chimie Physique, Université Paris Sud,

F-91405 Orsay Cedex, France

F. Spiegelman $(\varangle) \cdot$ N. Guihéry

Laboratoire de Chimie et Physique Quantiques,

Université Paul Sabatier, 118 route de Narbonne,

F-31062 Toulouse Cedex 9, France

E-mail: fernand.spiegelman@irsamc.ups-tlse.fr

J. Sanchez-Marin

Departament de Quimica-Fisica, Institut de Ciéncia Molecular,

Universitat de Valéncia, Dr. Moliner 50,

E-46100 Burjassot, Valencia, Spain able to give an example of a "general statement" in chemistry? etc. J.P. Malrieu was among the group and he was not the last to express firmly his views.

Between the sessions, P. Claverie, S. Diner and J. P. Malrieu had developed the PCILO method ("perturbative configuration interaction using localized bond orbitals"). Clearly, localized orbitals as well as perturbation theory are appealing tools whence you are interested in interpretation. In PCILO, they are combined with the CNDO approximation ("completely neglected differential overlap") where one assumes that any product of two different atomic orbitals can be treated as zero. An assumption that allows very simple formal developments and that provides a clear theoretical basis to such concepts as polarization, delocalization and various other types of bond interactions; but a very questionable assumption.

Actually, other fellows in the group had developed an ab initio approach (thus avoiding that assumption) using perturbation and localized bond orbitals. And they had found that the products of atomic orbitals are indeed important. For instance, all the nice bond interactions clarified in PCILO exactly cancel out in the case of the barrier to internal rotation in ethane molecule; only the effect of orbital products remains and it explains alone the value of the barrier! A similar result was found with the "W rule" concerning the stereospecificity of vicinal couplings in NMR and EPR ("nuclear magnetic resonance" and "electron paramagnetic resonance", two techniques which were emerging at that time). If two atoms are separated by three others forming a chain with a W shape, then the coupling is strong, otherwise it is small; a rule providing an extremely powerful tool to determine molecular structures. Actual ab initio calculations (using perturbation and localized bond orbitals) demonstrated that the $\mathrm{W}$ rule comes from compensation between through bond couplings, accounted for by PCILO and through space couplings neglected in CNDO.

And so, what about CNDO? - it is perfectly possible to explain all that using PCILO (and that was actually done), one has just to adjust the hopping parameter ...- of course a parameter! With enough parameters you fit an elephant! 
- or even a herd! Because PCILO is general, while using ab initio approaches requires to treat one case after the other ...- PCILO is general, but it provides reliable information on specific cases only if an experimental result is available to adjust the hopping parameter: what does it explain ?

Briefly, what is the issue of theory in Chemistry and Chemical Physics? Clearly a debate with no predictable end. Nevertheless, the debate was suddenly terminated during the years 1980-1990 when fast simulation programs became easily available (simulation including quantum chemistry as well as thermostatistics). Some comments on simulation versus theory would be useful here in the context of the Lagrasse meeting.

At first sight, what these simulation programs achieve essentially is just to provide an efficient tool for quantitative predictions. But a closer examination shows that the importance of such an achievement can hardly be overestimated. The key words here are "easily available" and "efficient" : what required a year of human efforts during the 1970s was achieved in a night by the computer in the 1980s. Thus, simulation had completely changed the current practice in most public and private chemical labs or services in Chemistry and Chemical Physics, and induced a complete renewal of the theoretical landscape. The nature of this renewal can be best seen by considering two examples.

The first example is provided by the description of $\mathrm{H}$-bonds in liquid water. The interaction energy between two water molecules can be analyzed as a sum of several terms (electrostatic, induction, charge transfer, exchange, dispersion, variation of internal correlation, etc.) ranging between -20 and $+20 \mathrm{~kJ} / \mathrm{mol}$ at equilibrium distance with a sum close to $-10 \mathrm{~kJ} / \mathrm{mol}$ (a smart analysis published by one of the fellows in 1974); accordingly, it appears necessary to analyze each of these terms separately to reach any qualitative conclusion; a difficult program, that can hardly be completed. Alternatively, one can just calculate the interaction energy and pass it (with no analysis) to a thermostatistics program. Then, new questions arise: what is the width of the attraction basin? What is that width important for? What is the influence of the environment ? etc. Answers: the basin is large with a narrow minimum; the narrow minimum is not important except for predicting the dielectric constant; the $\mathrm{N}$-atom interactions are critical for induction and an accurate value of the interaction energy in the dimer is neither sufficient nor necessary. Nobody cares if the basin is due to charge transfer or to exchange: such questions where important in a context where accurate predictions were just a dream; they become obsolete when the prediction can be performed "easily and efficiently".

Another example is related with the determination of structures of molecular ions in gas phase. Using basic theoretical chemistry one can determine a limited set of possible structures (usually, not just one) from the knowledge of how the ions have been obtained and which chemical reactions they do undergo. In order to choose among these structures, one can determine by simulation the vibrational spectrum of each one and compare with experiment (using for instance an "infra-red multiphoton dissociation" setup). And, except in case of very bad luck, a single structure comes out.

Thus, simulation rises new types of theoretical questions (first example), thus replacing other types of theoretical questions that become obsolete to some extent, but it does not replace basic theory (second example): it can be best understood not as a plain theory but as a bridge between theory and experiment and there is still a high demand on basic concepts (such as the ones used in the celebrated Marcus laws or Woodward-Hoffman rules) to be used as a guide for asking questions to simulations and experiment. And, in fact the practice of simulation has already lead to two important basic theoretical results (probably, not completely unexpected by some people, by now firmly established):

1. The electronic density in molecules is not much different from the simple juxtaposition of the densities of the constituent atoms; accordingly a simple interpolation procedure might be successful; and, in a sense, both the configuration interaction and the density functional approaches may be understood as interpolation procedures although most people would probably disagree: it comes from the fact that none of them can be used safely in a new and unique case; the success implies to first get some experience on similar problems which means that they are used (implicitly) as a comparison rather than a prediction method,

2. The structure of liquids (as characterized by the so-called $g(r)$ or "reduced distribution" functions) does not depend critically on the force field; accordingly, reasonable mean values can be obtained by simulations with a surprisingly small number of steps ${ }^{1}$ and using simple force fields should not be considered as a last resort to get a result that would be out of reach otherwise: on the contrary, it is a smart choice, perfectly justified by the nature of what is a liquid and certainly not to be completely changed as soon as computers run faster.

A subsidiary aspect of simulation is that it provides practical answers to most questions debated by our ten fellows around 1970 (what had not been found just by arguing): there is no need of theories that would replace numerical calculations (and in fact, no hope to find any), instead, theory and numerical calculations are both necessary; theory is required to develop basic concepts describing (or classifying or rationalizing) molecular processes what numerical calculations are unable to provide, but the practical counter parts of these concepts are no longer (or not only) algebraic formulas (contrary to the common practice all along the nineteenth and first-half of twentieth century) but prescriptions for the

\footnotetext{
${ }^{1}$ For instance, if the average number $n$ of steps per degree of freedom is defined by $n^{N}=N_{\text {step }}$ ( $N=$ number of degrees of freedom, $N_{\text {step }}=$ total number of steps in the simulation), then, typical values like $N=$ 1,000 and $N_{\text {step }}=10^{7}$, lead to $n=1.007$ ! A more common idea is that thermostatistics simulations are long and do not converge fast; still, they converge surprisingly fast by comparison with what could be expected assuming reasonable values like, say, $n=10$ steps per degree of freedom.
} 
appropriate simulation scheme; there is no issue in the question of using or avoiding adjustable parameters (ab initio vs. semi-empirical methods!) since all methods involve (explicitly or implicitly) adjustable parameters in some way (see above point 1); on the other hand, using a simulation scheme consistent with the considered physical or chemical problem is indeed critical.

The importance of simulation in Chemical Physics has been recognized by a Nobel prize in 1998 (Kohn and Pople). Of course, simulation does not result only from the work of these two outstanding scientists; many people were involved. Particularly, it may be viewed as resulting from a new and specific paradigm first outlined by Hartree (1939) and reformulated in a practical point of view by Boys and Metropolis around 1950. This paradigm is based on the idea that solving the equations describing a system (whatever the nature of that system is) with many variables is just impossible while predicting specific aspects using a computer might be possible if appropriate approximations are made (see the above points 1 and 2). The development of simulation in Chemical Physics proceeds from that paradigm. It was the start (or at least one of the important steps) of a general expansion of simulation that presently covers nearly all the fields of knowledge: nowadays, every one tries simulations in Physics, Biology, Physiology, Pharmacy, Neurology, Ecology, Economy, Social and Political Sciences. In view of the number and importance of its consequences, it is certainly a fundamental progress of science and probably a major one during the period 1950-2000.

In that context, the future might not be in Physics, Physical Chemistry, Biology and the like but in a new science studying simulation itself and using tools like the BayesLaplace probability theory to help in rationalizing the methods and extend the limits of simulation. On the whole, simulation appears as opening a new era and stimulating new exciting theoretical researches.

Of course, the outcome of a new era may imply that previous theoretical themes seem to become somehow obsolete (may be erroneously), which might appear to be frustrating and deserve negative reactions. But it is not something new that the advancement of knowledge results sometimes in unpleasant breaks. These breaks are stimulating for most people: it would be probably a mistake to describe science as a marvelous story that you can tell around in an effort to convince students to choose a science program rather than trade or law. It is likely that sciences appear more attractive if honestly described as a difficult attempt to improve knowledge with a risk to disturb the usual ways of thinking rather than a pleasant game where one wins at each trial.

At this point, it is fair and safe to apply this same critical point of view to simulation itself. Let us restrict here the arguments to molecular modeling, not excluding that some of the conclusions are extensible to other fields. Molecular modeling and simulation cover a variety of schemes. Let us mention a few of them with various ingredients, going from purely topological schemes (percolation schemes for instance) in which the shapes of the interaction poten- tials between particles are absent, molecular dynamics or Monte Carlo simulations using the very popular pair-additive Lennard Jones potentials, and finally the most sophisticated $a b$ initio packages. It is crucial to keep in mind that any of these schemes contains inherent more or less restrictive approximations, especially in concern with the interactions themselves and their transferability. If this is forgotten, there is a risk to generate a virtual reality, which while attractive and interesting, may unfortunately be misleading and poorly representative. Transferability is here a key question. For instance, do the properties of quasi-homogenous systems transpose to strongly heterogeneous systems? This point concerns several important fields. It is needed if one aims to derive reliable functionals in the context of Density Functional Theory. It is also relevant to understand whether models addressing pure water will actually transpose to solvation of strongly polar systems, possibly charged systems, possibly changing the hydrogen bonds networks structure and dynamics. It is also crucial to examine whether models or quantities reliable to describe given properties still hold for other ones. Is the $g(r)$ function the only relevant and sufficient observable to describe all the properties in liquids? What about fluctuations?

Precisely because of the increasing numerical efficiency of simulations and their associated graphical tools, allowing to derive strongly impacting pictures and scenarii, it is important today to emphasize that simulation is only a tool (toy?), hopefully approaching the physical or chemical truth, but certainly not to be confused with it. Any molecular simulation relies on a body of hypotheses, namely a constrained electronic interaction scheme associated with a dynamical processes, either stochastic or deterministic. Understanding and deriving those basic and elemental interactions and behavior still remains a fundamental challenge for Chemical Physics and Material Science, both qualitatively and quantitatively. The relevance of these questions remain even in the context of the very efficient ab initio packages, which are able to successfully address, either via Density Functional Theory, or via explicitly correlated methods, a variety of problems. Each scheme has a validity domain and in numerous cases, the description of electronic interactions still remains a challenge. Let us mention open shells highly correlated systems such as those encountered in molecular magnetism, so attractive now with the outburst of nanotechnologies and the perspective of addressing molecular spins individually or collectively (quantum information), transport properties in low dimensional systems or in proteins and the description of electron-phonon coupling, or also the determination of excited states and multiple-bond breaking in chemical reactions. There, theory is still needed.

It is a long-time dream that numerical simulation could help to design and tailor new materials, drugs or devices, investigating the possibility to make them functional, addressable and manipulable. In order to determine their final relevant properties and behavior, the usual scheme consists in first averaging the electrons degrees of freedom in the BornOppenheimer approximation and then averaging some or all 
the degrees of freedom of the nuclei over time or over phase space. Then, come questions which are still only poorly answered in usual large-scale simulations: are such averages valid and transferable and up to which space and time scales, what are the limitations of mean-field or mean-field like schemes and how can one extend and improve them? Those important questions bring us naturally back to the concept of fluctuations, and in the case of electrons, to the topic of correlation which remains a key concept. Electronic correlation has been the predilection field of J. P. Malrieu. So why not come back to our ten fellows before this text turns into a metaphysical lecture. Let us nevertheless mention that, beyond our search for efficiency and scientific productivity, more and more influenced by economical and societal evolution, such questions deserve to be debated.

After the year 1975 or so, the ten fellows were no longer working together and their interest had started to diverge. And what remains from their previous works, apart from contributions to the methodology of quantum chemistry and various specific results in chemical physics? One must realize that there have been general ideas behind the development of simulation, and it is likely that, explicitly or implicitly, PCILO was among these. Particularly, the ideas resulting from PCILO were used by one of the fellows (P. Claverie) to develop a systematic approach of inter-molecular forces. That approach had some success particularly in explaining the interactions between the bases of DNA (that was around 1973 and the situation was not as clear as it is now) and, in fact, many aspects developed in that approach are presently used in standard force fields. Thus, PCILO, a smart description of the electronic structure of molecules, was among the sources of the approaches that made the description of the electronic structure of molecules less critical. Let us just remember that it was among the sources, a remarkable achievement.

\section{And then in toulouse...}

PCILO was a semi-empirical method. However, while Quantum Chemistry up to the 1960s has been essentially the field of semi-empirical methods, the arrival of J.-P. Malrieu in Toulouse in 1974, corresponded to a period where the development of computer facilities made the early $a b$ initio methods, Hartree-Fock initially and very soon configuration interaction (CI) available for small systems, at least for the ground state. So this move occurred to be the conclusion of a period, and simultaneously the opening by J.-P. Malrieu and the subsequent newcomers of a new adventure. This adventure was based from the start and for many years, on a scientific and friendly everyday synergy with $\mathrm{Ph}$. Durand who had just gathered and oriented a small group of researchers towards quantum chemistry calculations. The "Laboratoire de Physique Quantique", newly created, then underwent an extremely fertile orientation around the combination of two main contributions brought together by $\mathrm{Ph}$. Durand and J.-P. Malrieu, respectively: pseudopotentials and configuration interaction. This combination actually opened one of the practical ways to the ab initio treatment of electronic structure in molecules. Pseudopotentials derived from the basic ideas of the chemical bond early formulated by Pauling became operational in computational quantum chemistry and gave access to the overall periodic table. Let us mention at this stage the interesting contribution of J.P. Malrieu for the treatment of core-valence correlation. Following the success of ab initio Hartree-Fock calculations, CI started to be developed in the 1970s. One of the favorite fields of J.P. Malrieu, if not the one, is certainly electron correlation. Before moving to Toulouse, he had just developed a very efficient idea with B. Huron and P. Rancurel, introducing a flexible scheme which combines variation and perturbation. They formulated and implemented a hierarchical CI scheme, by partitioning the numerous contributions into the larger ones requiring a variational treatment and the so-numerous less important ones which are perturbatively accounted for. This scheme named CIPSI (configuration interaction with perturbative selection through iterations) turned out to be numerically efficient for the electronic ground state. Furthermore, it opened very early the route to excited states, paralleling the only other code developed at that time by the German group of Bunker and Peyerimhoff (MRDCI). The applications were numerous essentially in two fields, chemistry (J.-C. Barthelat, G. Trinquier) certainly, but also in Molecular Physics (F. Spiegelman, F. X. Gadea,). Very quickly, the Toulouse tribe worked and grew around implementation and development, reinforced in 1979 by the arrival of J.-P. Daudey. The group was involved in laboratory and extra-laboratory regular meetings which turned out to be opportunities for numerous debates concerning science but also, from time to time, politics (perpetuating late in the night). Although not all speculations gave raise to operational tools, number of them did work and participated in the construction of that group and of its culture.

Thanks to its flexibility and generality, the methodology developed in CIPSI met a large success, yielding applications carried out by several groups, mainly in France, Italy and Spain. The CIPSI method clearly diverged from the brute force CI type methods based for instance on the unitary group, which were then dominant in Quantum Chemistry. It is interesting to notice now, more than 20 years later and after the Density Functional turn-over, that the same hierarchical basic concepts underlie most of the powerful methods today operational for explicit CI treatment of the excited states, such as CASPT2 or MRPT2 available in efficient quantumchemistry codes. It must be mentioned at this stage that this way of thinking goes beyond computational purposes. Indeed, J.-P. Malrieu has always been driven by the intention to understand the physical interactions governing the electronic structure in molecules, and later in more complex systems. As an example one may quote the analysis of the physical origin of symmetry breakings in mean-field (eventually multireference) calculations, and their possible use, for instance in the "intersticial description" of metals (M.-B. Lepetit, G. Durand). Each of the perturbative corrections can be understood as a well defined physical effect, 
possibly described by a diagram, often by hands and images. And many of the billions of indirect interactions can be mapped into effective interactions between a few leading configurations. The conceptual tool which allows such a condensation of information is the effective Hamiltonian theory, proposed by Bloch and des Cloizeaux. Thanks to this tool, it became possible to map complex ab initio calculations into simple descriptions and interpretations. This philosophy enabled theoreticians to interact with experimental chemists and molecular physicists, familiar with the single electron picture, in order to explain the subtle but sometimes crucial role of electronic correlation. Telling experimental chemists whether the electrons could be depicted as being in one or another electronic configuration, or even fluctuating between them, was not an easy task. Simultaneously, in the theoretical context, the effective hamiltonian scheme found some issues in consistent treatments of avoided crossings and state mixing, derivation of ab initio diabatic states and nonadiabatic couplings, consistent framework for hierarchical combination of electrostatics and spin-orbit coupling in CI (M. Pelissier and C. Teichteil).

In principle, the effective Hamiltonians, for instance in the quasi-degenerate perturbation theory, constitute a computational instrument for the treatment of multireference problems. Unfortunaely, the intruder states problem reduces severely its applicability. The elucidation of this problem has led J.-P. Malrieu, Ph. Durand and J.-P. Daudey to propose the intermediate effective Hamiltonian new object which made numerically operational the so-exciting intellectual idea of condensation of information. Not only this gave a tool to reduce the information, but also provided a way to address fundamental questions like size consistency and separability. The self-consistent dressing of CI matrices eliminating all unlinked contributions give raise to very efficient tools (J.-L. Heully, J.-P. Daudey, D. Maynau, N. Ben Amor, J. Sanchez Marin, M.B. Lepetit) the so-called Size-consistent self-consistent $(\mathrm{SC})^{2}$ method. The generalization to multireference problems (J. Meller, R. Caballol and N. Guihéry) happened to be the occasion to an intellectual partnership with D. Mukherjee, L. Meissner and U. Kaldor. The route was attractive, extremely fruitful, sometimes controversial, but after all rather efficient despite the number of paralleling but not-necessarily compatible versions issued by the codeveloper tribe in Toulouse and other collaborating people, sometimes generating headaches.

In the meantime, the experimental study of elementary reactions, namely atom-molecule and molecule-molecule had made a breakthrough with the development of sources and supersonic beam techniques. This field had built a bridge between chemical physics, quantum chemistry and molecular physics, formerly separate communities in France. Inducing the reaction, as well as analyzing the products could now be achieved via accurate laser techniques. Though J.-P. Malrieu has never been interested in dynamics, determination and description of multi-surface processes occurred to be a key point in various collaborations and provided a very fruitful application field of the methods developed in Toulouse (and also elsewhere) to address spectroscopy, photochemistry and photophysic issues, a field which was also fruitfully investigated by $\mathrm{B}$. Levy and $\mathrm{Ph}$. Millié. Let us remember that the knowledge of potential energy surfaces was extremely precious (and still is for small systems) at that period when the experimentalists knew everything before and after the reaction, but almost nothing during the chemical process itself, a rather frustrating expectation. This has considerably changed now with the development of time-resolved spectroscopy and femto-chemistry. For theoreticians, the challenge is still to deal with even more complex systems, such as nanoparticles, proteins or biological systems. Speaking about complexity, one must mention the study of clusters as another contact between F. Spiegelman, J. P. Malrieu and the Molecular Physics community (C. Bréchignac, M. Broyer, and P. Labastie who joined Toulouse in 1988). Indeed after monitoring reactive collisions, the experimentalists used their beams to continuously build small pieces of condensed matter unit by unit, from diatomics, to clusters, nano- and meso-particles up to micro-crystals. In clusters, the challenge of size is crucial, one of the key feature being quantum size effects. The capacity and care of J.P. Malrieu to develop on a parallel footing physical and computational aspects, that he was able to partly transmit to his coworkers, was of great help in designing consistent models, not completely general, but that could address situations in which ab initio calculations, CI certainly and even nowadays Density Functional just break down. Cluster physics has become the main topic of a full group in LPQ, actually after J.-P. Malrieu decided to orient his activity in other directions. After 1988, under the impulsion of J.-P. Malrieu, J.-P. Daudey and other coworkers, the competence of the Toulouse laboratory in addressing Molecular Physics questions, together with the motivation to increase the importance of fundamental physics and chemistry in Toulouse, has initiated the attraction of several experimental and theoretical groups, as well as individuals, in Toulouse. This operation finally gave birth to the "Institut de Recherche sur les Systèmes Atomiques et Moléculaires Complexes (IRSAMC)", institute which gathers now several laboratories and over 150 people.

\section{More recently....}

Despite his early interest for excited states, it is only more recently that J.-P. Malrieu entered, almost fortuitously, in the domain of magnetism. He was solicited by a group of experimentalists who had tried and failed to calculate the singlettriplet energy gap of a copper diacetate dimer using standard methods. Invoking multireference perturbative arguments, he proposed to calculate directly the energy difference between the two states. This approach implemented by J.-P. Daudey turned out to be successful and led him to propose later on the so-called Difference Dedicated Configuration Interaction (DDCI) method which is nowadays considered as the most reliable method of weak energy difference calculation in polyradicalar and magnetic systems. 
Simultaneously, his interest for valence bond interpretation of the effect of electronic correlation combined with the use of effective Hamiltonian theory led him and D. Maynau to propose a magnetic description of the conjugated hydrocarbon systems. From accurate ab initio calculations, they were able to extract a geometry-dependent Heisenberg Hamiltonian which happened to be an elegant interpretative tool and a predictive method for the study of both ground and excited states of organic conjugated molecules. The rather paradoxical relevance of this magnetic description of organic chemistry shows how fascinating and dangerous the frontier is between the strongly correlated (magnetic) and the strongly delocalized (covalent) domains. Also using a local description of ionic molecules, he proposed with F. X. Gadea the first $\mathrm{t}-\mathrm{J}$ effective Hamiltonian which became so famous, later on, for the study of high $T_{c}$ superconductors. Although these contributions did not receive a broad attention neither from chemists nor from physicists (despite the successful use of the related MMVB method by M. Robb in photochemistry), they marked the entrance of J.-P. Malrieu in the domain of strongly correlated fermions. This is a border field between physics and chemistry in which quantum descriptions cannot be circumvented. The physical chemists of this domain handle theoretical concepts when designing clever molecular systems and are wonderful intellectual partners for theoreticians. So was O. Kahn and so is M. Verdaguer of whom J.-P. Malrieu thinks highly.

While molecular magnetic systems of reasonable size can be addressed using quantum chemistry calculations, the understanding of collective properties of periodic lattices requires approximate treatment of model Hamiltonians. The production of J.-P. Malrieu in that peculiar field is twofold. A first practice which belongs to quantum chemistry consists in the determination of accurate model Hamiltonians. With both his spanish friends and collaborators R. Caballol, F. Illas, C. Jimenez Calzado, J. Sanz and some researchers of the Toulouse group, namely M.-B. Lepetit, N. Suaud and N. Guihéry, he developed a strategy for extracting model Hamiltonians that has resulted in an original and clever use of the effective Hamiltonian theory. Indeed, combining this theory with an accurate determination of the low-energy states of embedded fragments of solids, it becomes possible to validate the use of a model Hamiltonian and to extract its constitutive interactions. The quality of the extracted Hamiltonian strongly depends on the accuracy of the calculated low energy spectrum of the fragments. For this reason, a large part of his investment was devoted to methodological works and has resulted in the conception of efficient computational tools, the application range of which widely exceed the domain of magnetic systems. Let us mention for instance the several versions of DDCI which have been implemented by D. Maynau and several works on the determination of orbitals dedicated to an observable (R. Caballol). Looking for a less expensive multireference perturbative method, he also conceived with his italian partners C. Angeli and R. Cimiraglia the NEVPT2 method which eliminates unphysical intruder states through the use of a bielectronic zeroth-order Hamiltonian, following a former proposal by K. Dyall.

The second contribution of J.-P. Malrieu in the domain of strongly correlated fermions lies one step further in direction of solid state physics. During the last years, he developed theoretical methods inspired by his culture of quantum chemist with B. Miguel, M. Al Hajj, V. Robert and N. Guihéry. He has for instance proposed modified versions of Coupled Cluster Theory for the study of spin or electron periodic lattices. Let us mention finally the interesting proposition, the so-called Real Space Renormalization Group with effective interaction (RSRG-EI), which is an alternative to Density Matrix Renormalization Group (DMRG) that M.-B. Lepetit had already implemented in Toulouse. The RSRG-EI method combines the Wilson's wonderful idea of RSRG and the Bloch's effective Hamiltonian theory. This method presents the esthetics of Wilson's proposal which proceeds to an isomorphic scale change at each iteration and becomes numerically efficient. As well, the related Renormalized Excitonic method is another instrument for the study of the gaps in spin or electrons lattice and for the determination of quantum phase transitions.

These works have been the occasion of much collaboration between Spain, Italy and France. It is worth noting that J.-P. Malrieu plays a significant role in the south European cooperation often milestoned by pleasant meetings organized in wonderful sites. To satisfy his quest of esthetics, we were numerous (from time to time half dehydrated or soaked to the skin) to climb the pyrenean mountains through the most beautiful and inaccessible routes.

After this linear but non-exhaustive description of his scientific trajectory, let us give a few more general comments. J.-P. Malrieu serves numerous intellectual values in life, some of them being at times antagonist. As far as science is concerned, three harmoniously related ones have guided him along his scientific practice and illustrate faithfully his philosophy: formal rigor, predictive numerical efficiency and the production of qualitative interpretations.

To serve the former, J.-P. Malrieu proposed and still proposes methods and approximations physically relevant which give evidence of his formal and deep understanding of the many-body problem. Convinced that the computational challenge of quantum chemistry is not only technological but also logical, he entered the problem of electronic correlation and participated in most of the fights delivered by methodologists along the last decades. From the nightmares of intruder states to the fundamental question of size consistency, including the treatment of excited states as well as the treatment of collective effects in periodic lattices, rare are the topics related to electronic structure determination which he did not face up. It is also in this search for intelligibility that, with J.-P. Daudey and $\mathrm{Ph}$. Durand, he proposed the Intermediate Hamiltonian conceptual tool. Let us however mention that he did not get involved at all in the DFT methodologies. The present intervention of this method in the treatment of excited states as well as the recent trials of combinations between 
DFT and explicit correlation techniques lead us to think that J.-P. Malrieu should not have been so reluctant.

His methodological production testifies his care of providing predictive numerical efficiency. One should not understand in these words that he is able to parallelize a code. Far from it! Nevertheless, he has created predictive methods dedicated to the study of problems of chemical and/or physical interest, i.e., having a sufficiently wide application range. As well in the reference configuration selection as in the combination of variation and perturbation ("à la CIPSI or NEVPT2") or in the selection of excitations ("à la DDCI"), the goal is to scale the several physical effects in order to keep in the most expensive treatment only what is strictly needed. In the same spirit, one may also quote several works devoted to the determination of orbitals adapted to a given problem, like for instance dedicated orbitals or optimized localized orbitals (molecular orbitals or orthogonal atomic orbitals), the use of which leads to drastic reduction of the number of excitations that significantly contribute to electronic correlation. On this subject, one may find in the books of Chalvet et al. ${ }^{2}$ numerous ideas which are implemented nowadays in codes devoted to the treatment of electronic correlation using localized orbitals (see for instance his works with P. Reinhardt, A. Povill Battle, J. Rubio and the Toulouse group).

His care of producing efficient numerical methods is of course related to his practice in the domain of applications (which fatally require association with co-workers). From the first MP2 calculations performed on molecules with B. Levy to its very recent studies of magnetic systems, J.-P. Malrieu has always been interested in the physical interpretation of the electronic order and its changes. Often using the valence bond language, he likes to provide qualitative interpretations (as well as precise numbers) and metaphoric pictures allowing one to understand both the underlying physics and the role of electronic correlation. Let us mention, for instance, the orbital breathing while other simple and nice interpretations can be found in the rationalization of the polymorphism of insaturation (with G. Trinquier) and in the magnetic reading of conjugated organic chemistry (with D. Maynau). His will of producing qualitative picture, which he shares with R. Hoffmann and S. Shaik (and others...), is above all illustrated by his so-often call for the effective Hamiltonian theory which enables him to say: "Everything happens as if ..." and it is probably in the domain of magnetism that we may find his most famous uses of this efficient tool.

Since he did not wish to limit himself to a single and specific area, J.-P. Malrieu has developed a wide ranging and nevertheless highly consistent scientific activity. Deriving a deeper understanding of physics and chemistry from the study of complex electronic structure systems, he has managed to propose simultaneously more accurate and more efficient methods enabling to address more challenging systems. It is probably this singular interplay between a methodological practice and a real interest for both physics and chemistry that accounts for his remarkable intuition, which has resulted in such a high quality impacting scientific production.

2 "Localization and delocalization in quantum chemistry", Chalvet et al.(ed) Reidel, vols I and II, 1975 and 1976. 